\title{
Doppler factors, Lorentz factors and viewing angles for quasars, BL Lacertae objects and radio galaxies
}

\author{
T. Hovatta ${ }^{1}$, E. Valtaoja ${ }^{2,3}$, M. Tornikoski ${ }^{1}$, and A. Lähteenmäki ${ }^{1}$
}

\author{
${ }^{1}$ Metsähovi Radio Observatory, TKK, Helsinki University of Technology, Metsähovintie 114, 02540 Kylmälä, Finland \\ e-mail: tho@kurp.hut.fi \\ 2 Tuorla Observatory, University of Turku, Väisäläntie 20, 21500 Piikkiö, Finland \\ 3 Department of Physics and Astronomy, University of Turku, Vesilinnantie 5, 20100 Turku, Finland
}

Received 14 October 2008 / Accepted 18 November 2008

\begin{abstract}
Aims. We have calculated variability Doppler boosting factors, Lorentz factors, and viewing angles for a large sample of sources by using total flux density observations at 22 and $37 \mathrm{GHz}$ and VLBI data.

Methods. We decomposed the flux curves into exponential flares and determined the variability brightness temperatures of the fastest flares. By assuming the same intrinsic brightness temperature for each source, we calculated the Doppler boosting factors for 87 sources. In addition we used new apparent jet speed data to calculate the Lorentz factors and viewing angles for 67 sources. Results. We find that all quasars in our sample are Doppler-boosted and that the Doppler boosting factors of BL Lacertae objects are lower than of quasars. The new Lorentz factors are about twice as high as in earlier studies, which is mainly due to higher apparent speeds in our analyses. The jets of BL Lacertae objects are slower than of quasars. There are some extreme sources with very high derived Lorentz factors of the order of a hundred. These high Lorentz factors could be real. It is also possible that the sources exhibit such rapid flares that the fast variations have remained undetected in monitoring programmes, or else the sources have a complicated jet structure that is not amenable to our simple analysis. Almost all the sources are seen in a small viewing angle of less than 20 degrees. Our results follow the predictions of basic unification schemes for AGN.
\end{abstract}

Key words. galaxies: active - galaxies: jets - radio continuum: galaxies - radiation mechanisms: non-thermal galaxies: quasars: general

\section{Introduction}

All radio-bright active galactic nuclei (AGN) have relativistic jets emitting synchrotron radiation. The jets can at the simplest level be modelled by using two intrinsic parameters, the Lorentz factor $(\Gamma)$, which describes the speed of the jet flow, and the viewing angle $(\theta)$, which is the angle between the jet axis and the line of sight to the observer. These parameters can be calculated if the Doppler boosting factor $(D)$ and the apparent speed $\beta_{\text {app }}=v / c$ are known. We can find out the $\beta_{\text {app }}$ from Very Long Baseline Interferometry (VLBI) observations, and in the past few years major progress has been made in this area (e.g. Jorstad et al. 2001; Homan et al. 2001; Kellermann et al. 2004; Jorstad et al. 2005; Piner et al. 2007; Britzen et al. 2008). The Doppler boosting factors can be calculated in various ways, and different methods are compared in Lähteenmäki \& Valtaoja (1999, hereafter LV99).

A common way to calculate the Doppler boosting factors is to combine X-ray observations with VLBI component fluxes (e.g. Ghisellini et al. 1993; Guijosa \& Daly 1996; Guerra \& Daly 1997; Britzen et al. 2007). This method assumes inverse Compton (IC) origin of the X-ray emission, and that the same synchrotron photons forming the lower frequency radiation are responsible also for the IC emission. Assuming that the VLBI observations are done at the spectral turnover frequency, a predicted X-ray flux can be calculated. By comparing this to the observed X-ray flux, and by interpreting the excess flux as due to Doppler boosting, the Doppler boosting factors can be calculated. If the VLBI frequency is not at the turnover, large errors are induced in the Doppler boosting factors. This method also suffers greatly from non-simultaneous X-ray and VLBI data, and as was argued in LV99, gives much less accurate estimates for the Doppler boosting factors.

Using VLBI it is possible to directly observe the brightness temperature of the source $\left(T_{\mathrm{b}, \mathrm{obs}}\right)$. This can be compared to the intrinsic brightness temperature of the source $\left(T_{\mathrm{b}, \text { int }}\right)$, which is often assumed to be the equipartition temperature $\left(T_{\text {eq }}\right)$ (Readhead 1994; Lähteenmäki et al. 1999). The excess of $T_{\mathrm{b}, \mathrm{obs}}$ is interpreted as caused by Doppler boosting. This method also requires the values to be obtained at the turnover frequency, which enhances the errors in the Doppler boosting factors (LV99).

Another way to use VLBI observations is shown in Jorstad et al. (2005) who estimated the variability Doppler boosting factors of 15 AGN using Very Long Baseline Array (VLBA) data at $43 \mathrm{GHz}$. They calculated the flux decline time $\left(\tau_{\text {obs }} \propto \tau_{\text {int }} D\right)$ of a component in the jet and compared it to the measured size of the VLBI component (which does not depend on $D$ ). Assuming the intrinsic variability timescale corresponds to the light-travel time across the knot, they estimated the Doppler boosting factors. They also estimated the Lorentz factors and the viewing angles for these sources by using apparent speed data.

Variability timescales can also be obtained from total flux density (TFD) observations. This is the method used in LV99 and we use the same method in our analyses. We decompose each flux curve into exponential flares and calculate the variability timescale of each flare. From this we gain the observed 
brightness temperature, which is boosted by $D^{3}$ in comparison with $T_{\mathrm{b} \text {,int }}$. This makes possible the extraction of the variability Doppler factor $D_{\mathrm{var}}$ if $T_{\mathrm{b} \text {,int }}$ is known. In Lähteenmäki et al. (1999) it was argued, based on observations, that in every large flare $T_{\mathrm{b} \text {,int }}$ reaches the equipartition temperature $T_{\text {eq }}=5 \times 10^{10} \mathrm{~K}$.

In LV99 a sample of 81 sources was studied at 22 and $37 \mathrm{GHz}$ frequencies. They calculated the Doppler boosting factors based on observations from a period of over 15 years. For each source they combined the results of the two frequency bands and chose the fastest flare for the analysis. In addition they determined the Lorentz factors and the viewing angles for 45 sources. We have done similar calculations for a larger sample of sources and using data from almost 30 years of monitoring. We will also compare our results with the results of LV99 to see if the values have changed during the past 10 years.

The paper is organised as follows: in Sect. 2 we describe the source sample and the method used. In Sect. 3 we calculate the variability Doppler boosting factors and compare our results with LV99 and other related studies. The Lorentz factors and the viewing angles are presented in Sect. 4 and the discussion follows in Sect. 5. Finally conclusions are drawn in Sect. 6.

\section{Data and the method}

Our sample consists of 87 bright, well-monitored AGN from the Metsähovi Radio Observatory monitoring list. This is a rather good approximation of a complete flux limited sample of the brightest compact northern sources. (Missing are a handful of sources for which we had insufficient data to calculate the Doppler factors.) The sources have been observed regularly for almost 30 years at 22 and $37 \mathrm{GHz}$ with the Metsähovi $14 \mathrm{~m}$ telescope (Salonen et al. 1987; Teräsranta et al. 1992, 1998, 2004, 2005). Details of the observation method and data reduction process are described in Teräsranta et al. (1998). Our study also includes unpublished data at $37 \mathrm{GHz}$ from December 2001 until the end of 2006. Data of BL Lacertae objects at $37 \mathrm{GHz}$ from December 2001 until April 2005 are published in Nieppola et al. (2007). In our sample we have 30 high polarisation quasars (HPQs), which have optical polarisation exceeding 3 percent at some point in the past. In addition we have 22 low polarisation quasars (LPQs), 8 quasars (QSOs) for which no polarisation data were available, 22 BL Lacertae objects (BLOs), and 5 radio galaxies (GALs). We have used the classification used in the MOJAVE $^{1}$ programme whenever possible. All the quasars in our sample can be classified as flat spectrum radio quasars (FSRQs), which show blazar-like properties. Our sample does not contain ordinary quasars that have larger viewing angles and which do not show the rapid variability common for FSRQs. In our analyses we have usually combined QSOs with LPQs, following the choice of LV99. form

We decomposed the flux curves into exponential flares of the

$\Delta S(t)= \begin{cases}\Delta S_{\max } \mathrm{e}^{\left(t-t_{\max }\right) / \tau}, & t<t_{\max }, \\ \Delta S_{\max } \mathrm{e}^{\left(t-t_{\max }\right) / 1.3 \tau}, & t>t_{\max },\end{cases}$

where $\Delta S_{\max }$ is the maximum amplitude of the flare in janskys, $t_{\max }$ is the epoch of the flare maximum and $\tau$ is the rise time of the flare. The method is described in detail in Valtaoja et al. (1999). An example of a fit is presented in Fig. 1, where the flux curve of the HPQ source $1156+295$ is decomposed into exponential flares. The solid line represents the fitted sum curve

${ }^{1}$ http://www. physics. purdue.edu/MOJAVE/

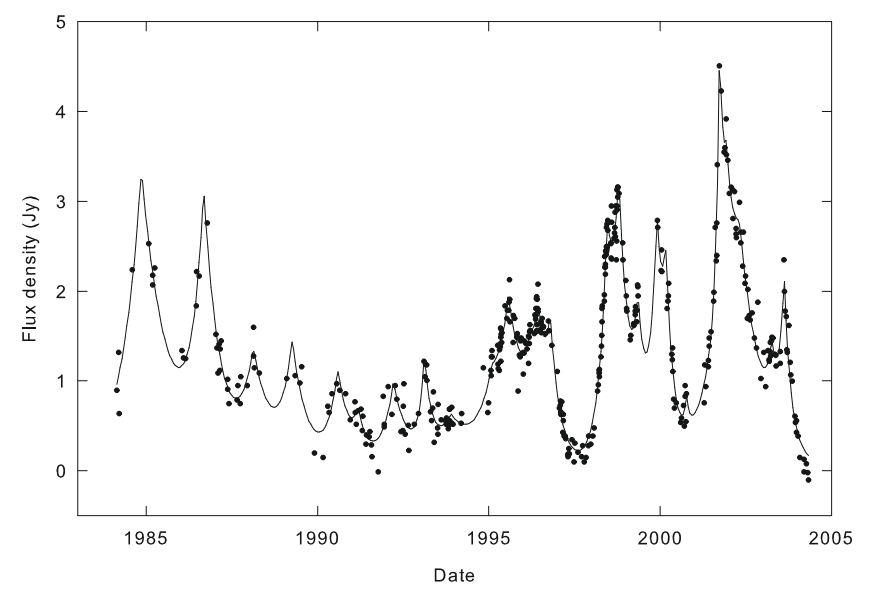

Fig. 1. Flux curve of the HPQ source 1156+295 (points) decomposed into exponential flares (solid line) at $22 \mathrm{GHz}$.

of the individual components and the points are actual observed data. We can see that the overall correspondence between the two is very good. In LV99 and Savolainen et al. (2002) it was also shown that the individual exponential flare components correspond very well to the emergence of new VLBI components, indicating that these flares obtained from the fits are indeed related to the actual jet physics.

From the fits we obtain the necessary parameters to calculate the observed variability brightness temperature of the source $T_{\mathrm{b} \text {,var }}$ (in the source proper frame)

$T_{\mathrm{b}, \mathrm{var}}=1.548 \times 10^{-32} \frac{\Delta S_{\max } d_{\mathrm{L}}^{2}}{v^{2} \tau^{2}(1+z)}$,

where $v$ is the observed frequency in $\mathrm{GHz}, z$ is the redshift, $d_{\mathrm{L}}$ is the luminosity distance in metres, and $\Delta S_{\max }$ and $\tau$ are defined in Eq. (1). The numerical factor in Eq. (2) corresponds to using $H_{0}=72 \mathrm{~km} \mathrm{~s}^{-1} \mathrm{Mpc}^{-1}, \Omega_{m}=0.27$ and $\Omega_{\Lambda}=0.73$, and to assuming that the source is a homogeneous sphere. In the calculation of the luminosity distances we have made use of the python version of the cosmology calculator created by Edward L. Wright ${ }^{2}$ (Wright 2006).

The variability Doppler factor can then be calculated as

$D_{\text {var }}=\left[\frac{T_{\mathrm{b}, \mathrm{var}}}{T_{\mathrm{b}, \text { int }}}\right]^{1 / 3}$

We used the value $T_{\mathrm{b} \text {,int }}=5 \times 10^{10} \mathrm{~K}$. This value was suggested by Readhead (1994) and the use of it was justified in Lähteenmäki et al. (1999). Based on simulations, Kellermann et al. (2004) also find the $T_{\mathrm{b} \text {,int }}$ to be of the order of $10^{11} \mathrm{~K}$.

By combining the Doppler boosting factors with apparent superluminal component velocities $\beta_{\text {app }}$, obtained using VLBI, we can calculate the variability Lorentz factors $\Gamma_{\text {var }}$ and the viewing angles $\theta_{\text {var }}$ by using Eqs. (4) and (5).

$\Gamma_{\mathrm{var}}=\frac{\beta_{\mathrm{app}}^{2}+D_{\mathrm{var}}^{2}+1}{2 D_{\mathrm{var}}}$

$\theta_{\mathrm{var}}=\arctan \frac{2 \beta_{\mathrm{app}}}{\beta_{\mathrm{app}}^{2}+D_{\mathrm{var}}^{2}-1}$.

We obtained $67 \beta_{\text {app }}$ values from the MOJAVE sample, observed with the VLBA at $15 \mathrm{GHz}$. The values are taken from the website

${ }^{2}$ http://www.astro.ucla.edu/ wright/CosmoCalc.html 
on September 9, 2008 and some of them may still be preliminary and change slightly in the final results (Lister et al. in preparation). This is the most homogeneous and largest sample of $\beta_{\text {app }}$ available at higher radio frequencies. These values represent the fastest reliable speed in each jet as measured by the MOJAVE programme at $15 \mathrm{GHz}$, using VLBA data spanning between 5 to 13 years, depending on the individual source.

\section{The variability Doppler boosting factors}

\subsection{Estimation of the Doppler boosting factors}

We were able to calculate the Doppler boosting factor $\left(D_{\mathrm{var}}\right)$ for 86 sources at $22 \mathrm{GHz}$, and for 72 sources at $37 \mathrm{GHz}$. For many sources we were able to determine the $D_{\text {var }}$ for more than one flare. The medians of $D_{\text {var }}$ are slightly larger at $22 \mathrm{GHz}$ than at $37 \mathrm{GHz}$, which could be due to different intrinsic brightness temperatures at the two frequency bands. In our analysis we chose the fastest flare of each source (either at 22 or $37 \mathrm{GHz}$, whichever had the fastest flare) to calculate the $D_{\text {var }}$, as was done in LV99. The argument for using the fastest flare to determine $D_{\mathrm{var}}$ is that they are most likely to reach the limiting brightness temperature and least likely to suffer from blending of flares which tends to increase the fitted timescale. This way we were able to calculate the $D_{\text {var }}$ for all the 87 sources in our sample. By visual examination, we divided the fits into three categories based on the goodness of the fit. No single numerical value, such as the $\chi^{2}$ test, is alone suitable for describing the goodness, because these usually characterise the entire flux curve, while we have only used one, fastest flare, to determine the $D_{\text {var }}$. In addition, relatively large error bars in some fainter sources cause the $\chi^{2}$ value to be small, while we consider a fit to be better when the scatter among the datapoints is small.

We classified the $D_{\text {var }}$ as excellent (E, 21 sources), good (G, 24 sources) or acceptable (A, 42 sources). In the fits classified as excellent, the exponential decomposition follows the datapoints quite precisely, as in the flares after the year 1997 in Fig. 1. The fit is also unambiguous and other functions do not describe the behaviour as well. Larger flares in Fig. 1 before 1995 would mainly be defined as good, because in these flares the fit follows the flux curve well, but there is also some scatter, and in some cases there are not as many datapoints to define the fit as in the ones defined as excellent. In the fits classified as acceptable, the scatter around the fit is still larger. This is often the case when the flux level of the source is modest and the errorbars consequently large. However, none of our results change significantly if we exclude the acceptable sources. All Doppler boosting factors are shown in Table 1 where the B1950-name, other commonly used name, type of the object, frequency of the $D_{\text {var }}$ determination, redshift, quality of the $D_{\text {var }}, \log T_{\mathrm{b}, \mathrm{var}}, D_{\mathrm{var}}, \beta_{\mathrm{app}}$, $\Gamma_{\mathrm{var}}, \theta_{\mathrm{var}}$, core dominance parameter $R$, and maximum optical polarisation $P_{\max }$ and its reference are listed.

It is difficult to determine exact error estimates for the $D_{\text {var }}$ of each source. Some indications can be obtained from the standard deviation of $D_{\text {var }}$ calculated from the various flares in one source. We calculated the deviations for all the sources classified as excellent, which had more than one flare to determine the $D_{\text {var }}$, including also flares determined as good. There were 45 such cases (including all the fits at both 22 and $37 \mathrm{GHz}$ ) and, on average, each source had 5.7 well-defined flares. The median standard deviation for these is $\sim 27 \%$, which can be thought of as an indication of the upper limit for the error estimate since in many cases the change in the $D_{\text {var }}$ of individual flares can also be due to differences in the source behaviour. It is also more likely to see a very fast flare in each source the longer they are monitored. More insight into the errors can be obtained when our new $D_{\text {var }}$ are compared to other studies (cf. Sect. 3.2).

In Table 2 we show the median values of $\log \left(T_{\mathrm{b}, \mathrm{var}}\right)$ and $D_{\mathrm{var}}$ for each source class separately and for HPQs and LPQs combined together (FSRQ). The distributions are shown in Figs. 2 and 3 and we can see that the distributions of quasars and BLOs have considerable overlap. HPQs have a tail extending to higher $D_{\text {var }}$ and LPQs seem to be in between the HPQs and BLOs. Also, it is interesting to note that all the quasars are clearly Doppler-boosted, the smallest estimated $T_{\mathrm{b} \text {,var }}$ being $3.5 \times 10^{11} \mathrm{~K}$ for $1928+738$. We ran the Kruskal-Wallis analysis to examine the differences between source classes. (All Kruskal-Wallis analyses in this paper have been performed with the Unistat statistical package for Windows ${ }^{3}$ (version 5.0).) The results confirm that all the source classes differ from the other classes significantly with a $95 \%$ confidence limit.

\subsection{Comparison with previous analyses}

Our sample has 71 sources in common with the sample of LV99. We have re-calculated the Doppler boosting factors of LV99, using the current cosmological model. Figure 4 shows the correlation between the Doppler boosting factors of LV99 and the new values. The results are very similar and the values correlate with a coefficient $r=0.77$ ( $p<0.0001)$. Kruskal-Wallis analysis also shows that the values come from the same population. This confirms that the method is reliable because the results have not changed even though we have now ten more years of data. The differences are mainly due to poor fits in LV99 which are due to poor sampling or low flux density (large scatter) in the data. The scatter between the old and the new values is consistent with the error analysis in Sect. 3.1. In some cases (e.g. 0430+052 and $1156+295)$ the source has clearly changed its behaviour and exhibits a much faster flare in our new dataset. The new estimates which are calculated using almost 30 years of data should therefore be more representative of the source behaviour.

We also compared our $D_{\text {var }}$ values with the Jorstad et al. (2005) values for 15 AGN, obtained at $43 \mathrm{GHz}$. Figure 5 shows the correlation between the two values, and a Spearman rank correlation gives a coefficient $r=0.56(p=0.0123)$. We can see that the values in Jorstad et al. (2005) are in general somewhat higher than ours. This can be due to their higher observing frequency (cf. Discussion). Also, their analysis method gives only an upper limit to some sources. The distribution of source classes is similar to ours, with quasars having the highest Doppler boosting factors, GALs the lowest and BLOs being in between them. Therefore we conclude that the Doppler boosting factors of these two analyses correspond well with each other.

Homan et al. (2006) argued that during the most active state $T_{\mathrm{b} \text {,int }}$ should be closer to $2 \times 10^{11} \mathrm{~K}$ and therefore the $D_{\text {var }}$ of the LV99 are overestimated. This would make our $D_{\text {var }}$ values even smaller, and the correspondence to Jorstad et al. (2005) would be worse. Higher $T_{\mathrm{b} \text {,int }}$ would also increase our Lorentz factors for the fastest sources, and as is shown later (cf. Sects. 4 and 5), our new values are already twice as high as in LV99 and in some sources even extremely high. We also note that a different value for $T_{\mathrm{b} \text {,int }}$ does not change the distributions themselves, only the numerical values.

We also compared our Doppler boosting factors to a recent study at a lower frequency of $5 \mathrm{GHz}$ (Britzen et al. 2007). They calculated the IC Doppler boosting factors by using VLBI

\footnotetext{
3 http: //WwW . unistat. com/
} 
Table 1. Doppler boosting factors, Lorentz factor and viewing angles for all sources.

\begin{tabular}{|c|c|c|c|c|c|c|c|c|c|c|c|c|c|}
\hline B1950-name & Other name & Type & $v$ & $z$ & Quality & $\log \left(T_{\mathrm{b}}\right)$ & $D_{\text {var }}$ & $\beta_{\text {app }}$ & $\Gamma_{\mathrm{var}}$ & $\theta_{\mathrm{var}}$ & $\log R$ & $P_{\max }$ & Ref. \\
\hline $0003-066$ & NRAO 5 & $\overline{B L O}$ & 22 & 0.347 & $\bar{A}$ & 12.82 & 5.1 & 2.660 & 3.3 & 9.5 & -0.133 & 3.4 & 11 \\
\hline $0007+106$ & III ZW 2 & GAL & 37 & 0.089 & $\mathrm{E}$ & 11.37 & 1.7 & 0.98 & 1.4 & 35.4 & 0.004 & 0.2 & 12 \\
\hline $0016+731$ & & LPQ & 37 & 1.781 & A & 13.40 & 7.9 & 6.67 & 6.8 & 7.1 & -0.104 & 0.6 & 11 \\
\hline 0048-097 & PKS 0048-097 & BLO & 22 & 0.300 & A & 13.65 & 9.6 & $\ldots$ & $\ldots$ & $\ldots$ & -0.082 & 27.1 & 2 \\
\hline $0059+581$ & & QSO & 22 & 0.644 & G & 14.15 & 14.1 & 11.1 & 11.5 & 4.0 & 0.018 & 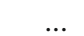 & $\ldots$ \\
\hline $0106+013$ & OC 012 & HPQ & 22 & 2.099 & $\mathrm{E}$ & 14.50 & 18.4 & 26.16 & 27.8 & 2.9 & -0.172 & 7.1 & 11 \\
\hline $0133+476$ & DA 55 & HPQ & 22 & 0.859 & G & 14.65 & 20.7 & 12.95 & 14.4 & 2.5 & -0.068 & 20.8 & 3 \\
\hline $0149+218$ & & LPQ & 37 & 1.320 & A & 12.83 & 5.1 & $\ldots$ & $\ldots$ & $\ldots$ & -0.024 & $\ldots$ & $\ldots$ \\
\hline $0202+149$ & 4C 15.05 & HPQ & 22 & 0.405 & $\mathrm{G}$ & 14.24 & 15.1 & 8.31 & 9.9 & 3.2 & -0.216 & 4.0 & 2 \\
\hline $0212+735$ & & HPQ & 22 & 2.367 & A & 13.48 & 8.5 & 7.35 & 7.5 & 6.7 & -0.268 & 7.8 & 2 \\
\hline $0219+428$ & $3 \mathrm{C} 66 \mathrm{~A}$ & BLO & 37 & 0.444 & A & 11.92 & 2.6 & $\ldots$ & $\ldots$ & $\ldots$ & & 29.7 & 5 \\
\hline $0224+671$ & & QSO & 22 & 0.523 & A & 13.43 & 8.2 & 11.67 & 12.5 & 6.6 & -0.308 & & $\ldots$ \\
\hline $0234+285$ & 4C 28.07 & HPQ & 37 & 1.213 & $\mathrm{G}$ & 14.32 & 16.1 & 12.23 & 12.7 & 3.4 & -0.090 & 11.3 & 2 \\
\hline $0235+164$ & & BLO & 22 & 0.940 & $\mathrm{G}$ & 14.84 & 24.0 & 2.000 & 12.1 & 0.4 & -0.092 & 44.7 & 2 \\
\hline $0306+102$ & PKS $0306+102$ & BLO & 22 & 0.863 & A & 13.97 & 12.3 & & & & & & $\ldots$ \\
\hline $0316+413$ & $3 \mathrm{C} 84$ & GAL & 37 & 0.018 & G & 9.26 & 0.3 & 0.32 & 1.8 & 39.1 & -0.654 & 6.0 & 11 \\
\hline $0333+321$ & NRAO 140 & LPQ & 22 & 1.263 & G & 14.74 & 22.2 & 12.7 & 14.7 & 2.2 & -0.140 & 0.7 & 12 \\
\hline 0336-019 & CTA 026 & HPQ & 22 & 0.852 & A & 14.42 & 17.4 & 22.32 & 23.0 & 3.2 & -0.133 & 19.4 & 2 \\
\hline $0355+508$ & NRAO 150 & QSO & 37 & 1.510 & G & 14.05 & 13.1 & & & & -0.015 & $\ldots$ & $\ldots$ \\
\hline $0415+379$ & $3 \mathrm{C} 111$ & GAL & 37 & 0.049 & G & 12.09 & 2.9 & 5.94 & 7.7 & 15.5 & -0.432 & 3.1 & 5 \\
\hline 0420-014 & OA 129 & HPQ & 37 & 0.915 & $\mathrm{E}$ & 14.59 & 19.9 & 7.52 & 11.4 & 1.9 & -0.097 & 26.2 & 11 \\
\hline $0430+052$ & $3 \mathrm{C} 120$ & GAL & 22 & 0.033 & G & 13.01 & 5.9 & 5.42 & 5.5 & 9.7 & -0.488 & 0.5 & 5 \\
\hline $0440-003$ & NRAO 190 & HPQ & 22 & 0.844 & A & 14.03 & 12.9 & $\ldots$ & $\ldots$ & $\ldots$ & -0.291 & 12.5 & 11 \\
\hline $0458-020$ & PKS 0458-020 & HPQ & 37 & 2.286 & $\mathrm{G}$ & 14.29 & 15.8 & 16.2 & 16.2 & 3.6 & -0.134 & 17.3 & 2 \\
\hline $0528+134$ & PKS 0528+134 & HPQ & 22 & 2.070 & $\mathrm{E}$ & 15.18 & 31.2 & 18.73 & 21.2 & 1.6 & -0.059 & 4.1 & 5 \\
\hline $0552+398$ & DA 193 & LPQ & 37 & 2.363 & A & 14.91 & 25.2 & 0.45 & 12.6 & 0.1 & -0.157 & 1.9 & 12 \\
\hline $0605-085$ & PKS 0605-085 & HPQ & 37 & 0.872 & A & 13.34 & 7.6 & 19.98 & 30.2 & 5.0 & -0.191 & 9.8 & 11 \\
\hline $0642+449$ & $\mathrm{OH} 471$ & LPQ & 22 & 3.396 & $\mathrm{G}$ & 13.78 & 10.7 & 0.53 & 5.4 & 0.5 & -0.192 & 1.7 & 7 \\
\hline $0716+714$ & & BLO & 22 & 0.310 & $\mathrm{E}$ & 13.81 & 10.9 & 10.22 & 10.3 & 5.2 & -0.219 & 17.3 & 4 \\
\hline $0735+178$ & PKS $0735+17$ & BLO & 37 & 0.424 & G & 12.43 & 3.8 & & & & -0.378 & 36.0 & 2 \\
\hline $0736+017$ & & HPQ & 22 & 0.191 & $\mathrm{E}$ & 13.50 & 8.6 & 14.73 & 17.0 & 5.8 & -0.157 & 8.4 & 11 \\
\hline $0754+100$ & OI 090.4 & BLO & 37 & 0.266 & A & 12.95 & 5.6 & 14.530 & 21.7 & 6.9 & -0.168 & 26.0 & 1 \\
\hline $0804+499$ & & HPQ & 22 & 1.436 & $\mathrm{E}$ & 15.35 & 35.5 & 1.81 & 17.8 & 0.2 & -0.096 & 8.6 & 2 \\
\hline $0814+425$ & & BLO & 22 & 0.245 & $\mathrm{G}$ & 12.69 & 4.6 & 1.720 & 2.7 & 8.4 & -0.139 & 8.7 & 2 \\
\hline $0827+243$ & OJ 248 & LPQ & 37 & 0.941 & A & 14.05 & 13.1 & 21.3 & 23.9 & 3.9 & -0.037 & 1.5 & 7 \\
\hline $0836+710$ & 4C 71.07 & LPQ & 37 & 2.218 & A & 14.33 & 16.3 & 25.39 & 28.0 & 3.2 & -0.119 & 1.1 & 11 \\
\hline $0847-120$ & J 0850-1213 & QSO & 37 & 0.566 & G & 14.35 & 16.5 & & & & & & $\ldots$ \\
\hline $0851+202$ & OJ 287 & BLO & 22 & 0.306 & E & 14.39 & 17.0 & 15.31 & 15.4 & 3.3 & -0.181 & 15.6 & 5 \\
\hline $0923+392$ & 4C 39.25 & LPQ & 37 & 0.695 & G & 12.60 & 4.3 & 42.94 & 216.1 & 2.6 & -1.757 & 0.5 & 2 \\
\hline $0945+408$ & $4 C 40.24$ & HPQ & 37 & 1.249 & A & 13.13 & 6.4 & 18.47 & 29.8 & 5.5 & -0.274 & 6.5 & 3 \\
\hline $0953+254$ & & LPQ & 37 & 0.712 & A & 12.61 & 4.3 & $\ldots$ & $\ldots$ & $\ldots$ & -0.268 & 2.2 & 2 \\
\hline $0954+658$ & S4 0954+65 & BLO & 37 & 0.367 & A & 13.07 & 6.2 & $\ldots$ & $\ldots$ & $\ldots$ & -0.054 & 33.7 & 11 \\
\hline $1055+018$ & OL 093 & HPQ & 37 & 0.888 & G & 13.96 & 12.2 & 10.96 & 11.1 & 4.7 & -0.159 & 5.0 & 2 \\
\hline $1156+295$ & 4C 29.45 & HPQ & 22 & 0.729 & $\mathrm{E}$ & 15.06 & 28.5 & 24.85 & 25.1 & 2.0 & -0.080 & 9.2 & 12 \\
\hline $1219+285$ & ON 231 & BLO & 22 & 0.102 & A & 10.96 & 1.2 & $\ldots$ & $\ldots$ & $\ldots$ & -0.363 & 10.0 & 1 \\
\hline $1222+216$ & PKS $1222+216$ & LPQ & 37 & 0.432 & A & 12.85 & 5.2 & 21.12 & 45.5 & 5.1 & -0.210 & 1.5 & 9 \\
\hline $1226+023$ & $3 \mathrm{C} 273$ & LPQ & 22 & 0.158 & $\mathrm{E}$ & 14.39 & 17.0 & 13.59 & 14.0 & 3.3 & -0.809 & 0.5 & 2 \\
\hline $1253-055$ & $3 C 279$ & HPQ & 37 & 0.536 & $\mathrm{E}$ & 14.84 & 24.0 & 20.64 & 20.9 & 2.4 & -0.242 & 39.2 & 5 \\
\hline $1308+326$ & AU CV n & HPQ & 22 & 0.997 & $\mathrm{G}$ & 14.26 & 15.4 & 27.500 & 32.2 & 3.2 & -0.096 & 25.0 & 2 \\
\hline $1324+224$ & & QSO & 22 & 1.400 & A & 14.68 & 21.2 & 3.04 & 10.9 & 0.8 & -0.075 & $\ldots$ & $\ldots$ \\
\hline $1413+135$ & & BLO & 22 & 0.247 & G & 13.96 & 12.2 & 1.860 & 6.3 & 1.4 & -0.112 & 13.0 & 8 \\
\hline $1418+546$ & OQ 530 & BLO & 22 & 0.151 & A & 12.83 & 5.1 & & $\ldots$ & $\ldots$ & -0.175 & 19.0 & 1 \\
\hline $1502+106$ & OR 103 & HPQ & 37 & 1.839 & $\mathrm{E}$ & 13.94 & 12.0 & 14.6 & 14.9 & 4.7 & -0.202 & 3.0 & 2 \\
\hline $1510-089$ & PKS 1510-089 & HPQ & 37 & 0.360 & $\mathrm{E}$ & 14.37 & 16.7 & 20.29 & 20.7 & 3.4 & -0.167 & 7.8 & 11 \\
\hline $1538+149$ & 4C 14.60 & BLO & 37 & 0.605 & A & 12.60 & 4.3 & 8.750 & 11.2 & 10.5 & -0.296 & 17.4 & 2 \\
\hline $1606+106$ & 4C 10.45 & LPQ & 22 & 1.226 & $\mathrm{G}$ & 14.89 & 25.0 & 18.78 & 19.6 & 2.2 & -0.081 & 1.9 & 11 \\
\hline $1611+343$ & DA 406 & LPQ & 37 & 1.401 & A & 14.11 & 13.7 & 14.17 & 14.2 & 4.2 & -0.179 & 2.3 & 6 \\
\hline $1633+382$ & $4 C 38.41$ & HPQ & 22 & 1.814 & $\mathrm{E}$ & 14.70 & 21.5 & 29.13 & 30.5 & 2.5 & -0.256 & 7.0 & 6 \\
\hline $1637+574$ & OS 562 & LPQ & 22 & 0.751 & A & 14.13 & 14.0 & 10.61 & 11.0 & 4.0 & -0.093 & 1.5 & 12 \\
\hline $1641+399$ & $3 \mathrm{C} 345$ & HPQ & 37 & 0.593 & $\mathrm{E}$ & 13.38 & 7.8 & 19.27 & 27.7 & 5.1 & -0.412 & 38.3 & 5 \\
\hline $1725+044$ & PKS $1725+044$ & QSO & 22 & 0.293 & A & 12.44 & 3.8 & & & & & $\ldots$ & $\ldots$ \\
\hline $1730-130$ & NRAO 530 & QSO & 37 & 0.902 & $\mathrm{G}$ & 13.79 & 10.7 & 35.6 & 64.6 & 3.0 & -0.291 & $\ldots$ & $\ldots$ \\
\hline $1739+522$ & S4 1739+52 & HPQ & 22 & 1.375 & $\mathrm{E}$ & 14.97 & 26.5 & $\ldots$ & $\ldots$ & $\ldots$ & -0.040 & 3.7 & 11 \\
\hline $1741-038$ & PKS 1741-038 & $\mathrm{HPQ}$ & 22 & 1.054 & $\mathrm{E}$ & 14.58 & 19.7 & & $\ldots$ & $\cdots$ & -0.055 & 9.2 & 2 \\
\hline $1749+096$ & PKS 1749+096 & BLO & 37 & 0.322 & $\mathrm{E}$ & 13.93 & 12.0 & 5.880 & 7.5 & 3.8 & -0.030 & 31.3 & 2 \\
\hline $1803+784$ & S5 1803+784 & BLO & 22 & 0.684 & A & 13.96 & 12.2 & 8.980 & 9.5 & 4.5 & -0.250 & 7.0 & 3 \\
\hline
\end{tabular}


Table 1. continued.

\begin{tabular}{llllllllrrrrrr}
\hline \hline B1950-name & Other name & Type & $v$ & $z$ & Quality & $\log \left(T_{\mathrm{b}}\right)$ & $D_{\text {var }}$ & $\beta_{\text {app }}$ & $\Gamma_{\text {var }}$ & $\theta_{\text {var }}$ & $\log R$ & $P_{\max }$ & Ref. \\
\hline $1807+698$ & 3C 371.0 & BLO & 37 & 0.051 & $\mathrm{~A}$ & 10.78 & 1.1 & 0.120 & 1.0 & 57.3 & -0.328 & 8.0 & 3 \\
$1823+568$ & 4C 56.27 & BLO & 22 & 0.663 & $\mathrm{~A}$ & 13.11 & 6.4 & 20.950 & 37.8 & 5.0 & -0.154 & 29.8 & 5 \\
$1828+487$ & 3C 380 & LPQ & 37 & 0.692 & $\mathrm{~A}$ & 12.97 & 5.7 & 13.66 & 19.3 & 7.1 & -0.391 & 0.4 & 2 \\
$1928+738$ & 4C 73.18 & LPQ & 22 & 0.302 & $\mathrm{~A}$ & 11.55 & 1.9 & 8.48 & 19.9 & 12.8 & -0.402 & 0.8 & 2 \\
$1954+513$ & & LPQ & 22 & 1.223 & $\mathrm{~A}$ & 13.30 & 7.4 & $\ldots$ & $\ldots$ & $\ldots$ & -0.181 & 1.4 & 11 \\
$2005+403$ & & QSO & 37 & 1.736 & $\mathrm{~A}$ & 14.07 & 13.3 & 19.48 & 21.0 & 4.0 & -0.257 & $\ldots$ & $\ldots$ \\
$2007+776$ & S5 2007+77 & BLO & 22 & 0.342 & $\mathrm{~A}$ & 13.39 & 7.9 & $\ldots$ & $\ldots$ & $\ldots$ & -0.304 & 15.1 & 11 \\
$2021+614$ & OW 637 & GAL & 22 & 0.227 & $\mathrm{~A}$ & 11.65 & 2.1 & 0.41 & 1.3 & 13.3 & $\ldots$ & 0.3 & 3 \\
$2022+171$ & & LPQ & 22 & 1.050 & $\mathrm{~A}$ & 12.99 & 5.8 & $\ldots$ & $\ldots$ & $\ldots$ & $\ldots$ & $\ldots$ & $\ldots$ \\
$2121+053$ & & HPQ & 22 & 1.941 & $\mathrm{~A}$ & 14.25 & 15.3 & 13.05 & 13.2 & 3.7 & -0.061 & 10.7 & 7 \\
$2134+004$ & OX 057 & LPQ & 22 & 1.932 & $\mathrm{~A}$ & 14.32 & 16.1 & 5.62 & 9.0 & 2.2 & -0.405 & 2.8 & 12 \\
$2136+141$ & & LPQ & 22 & 2.427 & $\mathrm{~A}$ & 13.46 & 8.3 & 5.08 & 5.8 & 6.2 & -0.175 & 1.6 & 11 \\
$2145+067$ & & LPQ & 37 & 0.990 & $\mathrm{G}$ & 14.28 & 15.6 & 2.49 & 8.0 & 1.1 & -0.171 & 0.6 & 2 \\
$2200+420$ & BL LAC & BLO & 37 & 0.069 & $\mathrm{E}$ & 13.28 & 7.3 & 10.700 & 11.6 & 7.3 & -0.264 & 23.0 & 1 \\
$2201+315$ & 4C 31.63 & LPQ & 22 & 0.295 & $\mathrm{G}$ & 13.17 & 6.7 & 7.88 & 8.1 & 8.5 & -0.096 & 0.2 & 2 \\
$2223-052$ & 3C 446 & HPQ & 22 & 1.404 & $\mathrm{G}$ & 14.31 & 16.0 & 16.470 & 16.5 & 3.6 & -0.203 & 17.3 & 10 \\
$2227-088$ & & HPQ & 22 & 1.562 & $\mathrm{~A}$ & 14.31 & 15.9 & 4.95 & 8.8 & 2.0 & -0.137 & 6.9 & 12 \\
$2230+114$ & CTA 102 & HPQ & 37 & 1.037 & $\mathrm{E}$ & 14.28 & 15.6 & 15.51 & 15.5 & 3.7 & -0.269 & 10.9 & 7 \\
$2234+282$ & & HPQ & 22 & 0.795 & $\mathrm{~A}$ & 13.03 & 6.0 & $\ldots$ & $\ldots$ & $\ldots$ & -0.464 & 4.4 & 11 \\
$2251+158$ & 3C 454.3 & HPQ & 37 & 0.859 & $\mathrm{E}$ & 15.26 & 33.2 & 14.86 & 19.9 & 1.3 & -0.564 & 16.0 & 1 \\
$2254+074$ & PKS 2254+074 & BLO & 22 & 0.190 & $\mathrm{~A}$ & 11.91 & 2.5 & $\ldots$ & $\ldots$ & $\ldots$ & $\ldots$ & 21.0 & 1 \\
\hline
\end{tabular}

References: (1) Angel \& Stockman (1980); (2) Impey \& Tapia (1990); (3) Impey et al. (1991); (4) Impey et al. (2000); (5) Jorstad et al. (2007); (6) Lister \& Smith (2000); (7) Moore \& Stockman (1984); (8) Punsly (1996); (9) Sluse et al. (2005); (10) Smith et al. (1985); (11) Stickel \& Kuehr (1994); (12) Wills et al. (1992).

Table 2. Median values of $\log \left(T_{\mathrm{b}, \mathrm{var}}\right)$ and $D_{\mathrm{var}}$.

\begin{tabular}{lccc}
\hline \hline Type & $N$ & $\log \left(T_{\text {b,var }}\right)[\mathrm{K}]$ & $D_{\text {var }}$ \\
\hline HPQ & 30 & 14.31 & 15.98 \\
LPQ & 30 & 13.92 & 11.90 \\
FSRQ & 60 & 14.19 & 14.61 \\
BLO & 22 & 13.09 & 6.25 \\
GAL & 5 & 11.65 & 2.07 \\
ALL & 87 & 13.94 & 12.02 \\
\hline
\end{tabular}

data from the Caltech-Jodrell Bank Flat-Spectrum source sample (Taylor et al. 1996) and non-simultaneous ROSAT X-ray data. The Spearman rank correlation between our 24 common sources (excluding one outlier, $0836+710$, with $D_{\text {IC }}=88$ ) is still quite $\operatorname{good}(r=0.63, p=0.0004)$, and the slope of the linear fit between $D_{\mathrm{IC}}$ and $D_{\mathrm{var}}$ is almost exactly one. We believe that most of the scatter is due to the errors in the IC Doppler boosting factors, since LV99 showed that for several reasons these are likely to be much less accurate than the variability Doppler boosting factors.

\subsection{Core dominance}

Standard beaming models expect that more core-dominated objects should be more beamed and thus have higher Doppler boosting factors. We studied this by calculating the coredominance parameter $R$ from VLBA data of Kovalev et al. (2005) at $15 \mathrm{GHz}$. The core-dominance is calculated by relating the flux density of the core $S_{\text {core }}$ to the total single-dish flux density observed at $15 \mathrm{GHz} S_{\text {tot }}$. In Kovalev et al. (2005) these are given for 250 sources observed with the VLBA at $15 \mathrm{GHz}$ at different epochs. Their sample includes 80 sources for which we have determined $D_{\text {var }}$. We calculated the median $R$ for each source from the separate epochs. Figure 6 shows the correlation between $\log R$ and $\log D_{\text {var }}$, excluding an outlier

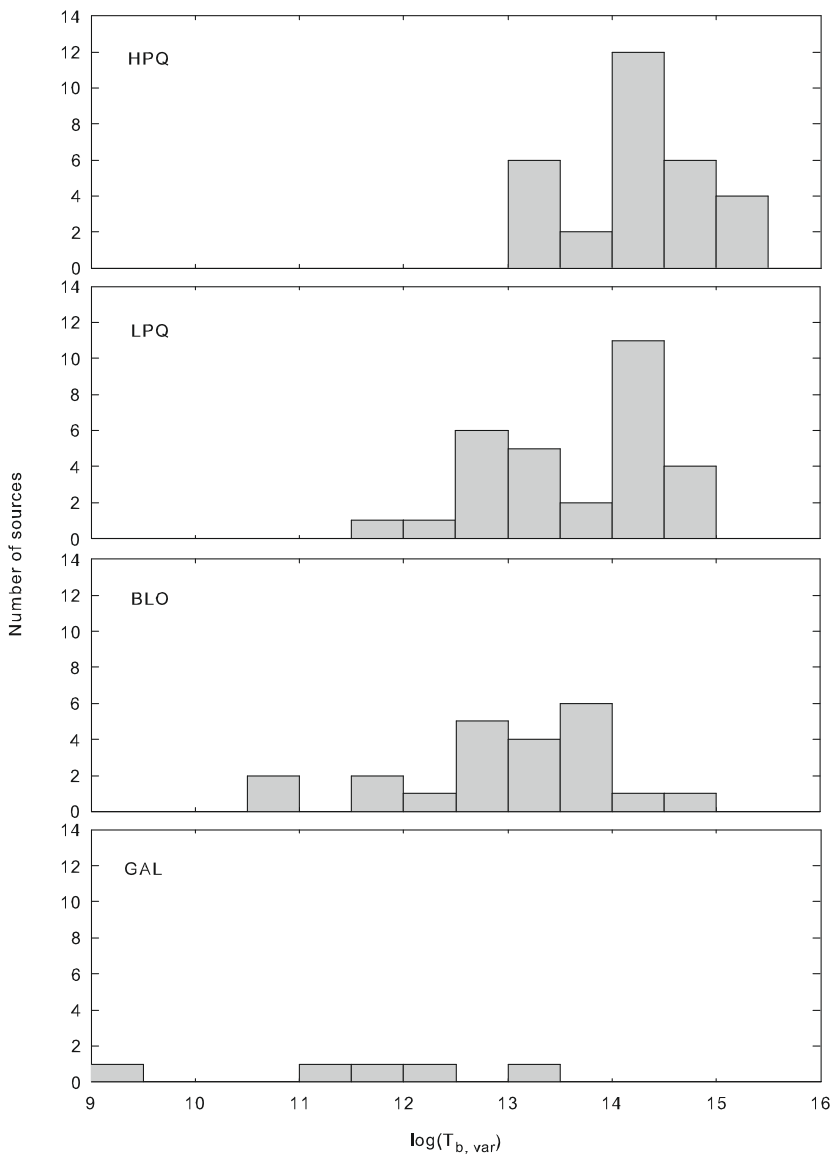

Fig. 2. Distribution of $T_{\mathrm{b}, \text { var }}$ of the fastest flare in each source.

Source $0923+392$ with a very small core dominance $(\log R=$ $-1.76)$. Spearman rank correlation between the parameters is $r=0.37(p=0.0004)$. When the outlier source is included, the 


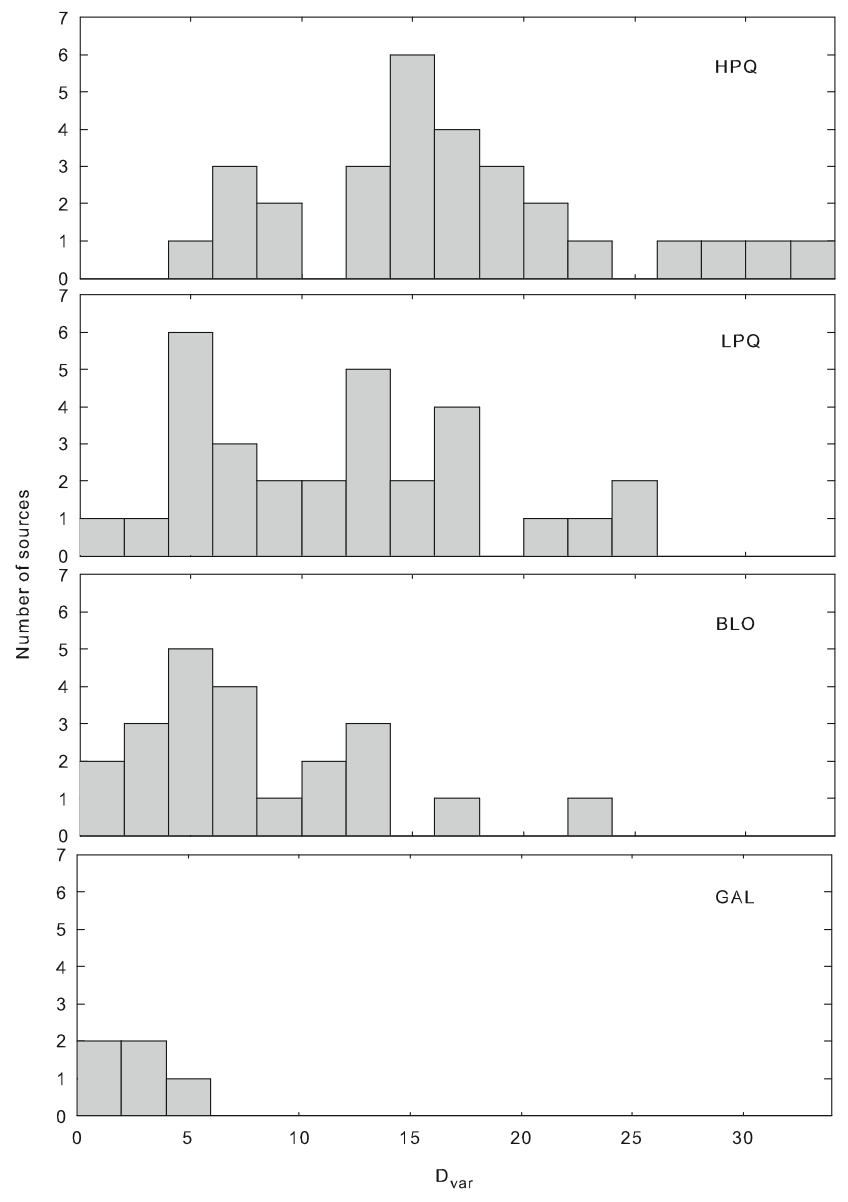

Fig. 3. Distribution of $D_{\mathrm{var}}$ of the fastest flare in each source.

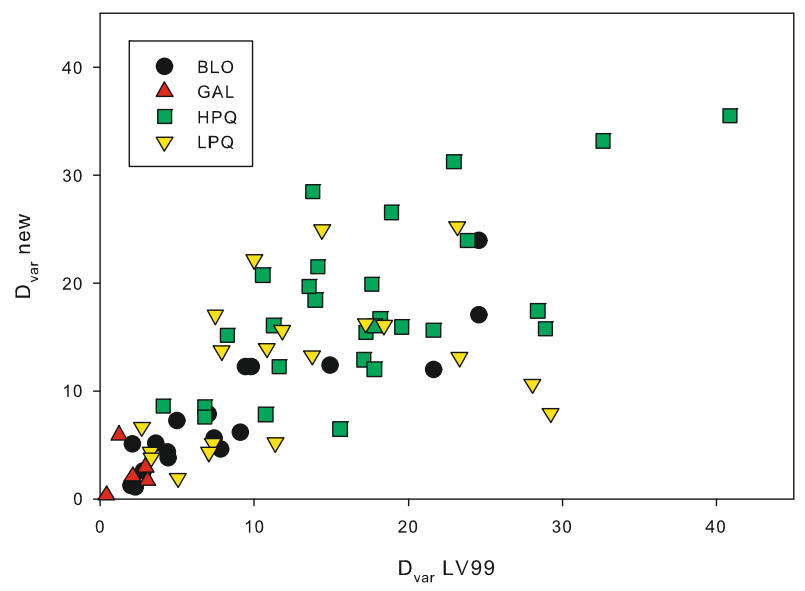

Fig. 4. Correlation between the $D_{\text {var }}$ from LV99 and our new $D_{\text {var }}$ values.

correlation is still significant with a coefficient $r=0.39(p=$ 0.0002). This shows that there is indeed some indication that sources which are more core-dominated are also more boosted. In Kovalev et al. (2005) the core-dominance is defined to be the relation of $S_{\text {core }}$ to the total VLBA flux density $S_{\text {VLBA. }}$. We tested the correlation using also this parameter but the results did not change because $S_{\text {tot }}$ and $S_{\mathrm{VLBA}}$ are so similar. Using the coredominance defined with $S_{\mathrm{VLBA}}$, Kovalev et al. (2005) show that quasars and BLOs are significantly different from GALs with lower core-dominance.

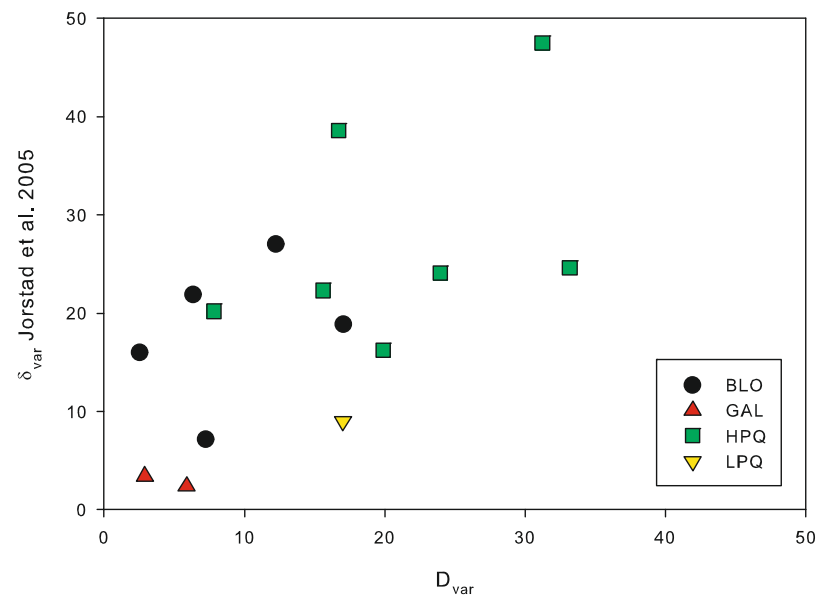

Fig. 5. Correlation between the $D_{\text {var }}$ and $\delta_{\text {var }}$ from Jorstad et al. (2005).

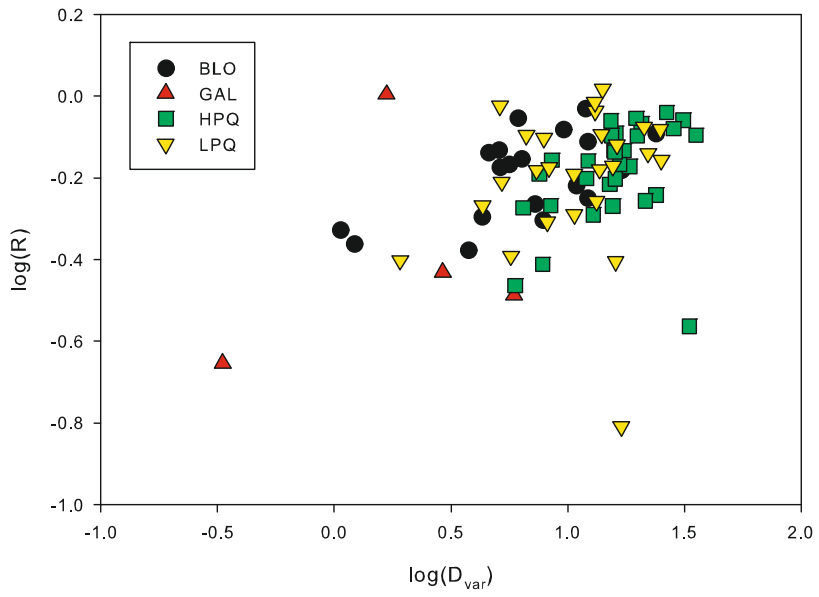

Fig. 6. Correlation between $\log (R)$ using data from Kovalev et al. (2005) and $\log \left(D_{\text {var }}\right)$, excluding the outlier source $0923+392$ with $\log (R)=$ -1.76 and $\log \left(D_{\text {var }}\right)=0.63$.

Similar calculations were made in Britzen et al. (2007) for their sample. They used the core flux density and total singledish flux density at $5 \mathrm{GHz}$ to calculate the core-dominance parameter. They found no significant correlation between their core-dominance parameter and the IC Doppler boosting factor. We have only 25 sources in common with their sample, and when we compared our $D_{\text {var }}$ with their core-dominance parameter, we found no correlation.

\section{The Lorentz factors and viewing angles}

We were able to calculate Lorentz factors $\Gamma_{\text {var }}$ and viewing angles $\theta_{\text {var }}$ for 67 sources. The median values of different source classes are shown in Table 3. These are affected by two outliers with exceptionally large $\Gamma_{\text {var }}$. The source $0923+392$ has $\Gamma_{\text {var }}=216.1$ and $1730-130$ has $\Gamma_{\text {var }}=64.6$. Both of these show high superluminal motion of $\beta_{\text {app }}>35 c$, which increases the Lorentz factors. The distributions of the source classes are shown in Figs. 7 and 8. In $\Gamma_{\text {var }}$ the distributions of quasars and BLOs overlap, but BLOs and GALs have slower jet speeds than quasars. Kruskal-Wallis analysis shows that when $\Gamma_{\text {var }}$ are studied without the outlier sources, HPQs differ from other classes with higher Lorentz factors, and GALs differ with smaller Lorentz factors. BLOs and LPQs come from the same population with a $13 \%$ confidence. There is one BLO $(1823+568)$ 
Table 3. Median values of $\Gamma_{\text {var }}$ and $\theta_{\text {var }}$.

\begin{tabular}{lrrr}
\hline \hline Type & $N$ & $\Gamma_{\text {var }}$ & $\theta_{\text {var }}$ \\
\hline HPQ & 26 & 17.41 & 3.28 \\
LPQ & 23 & 13.96 & 3.90 \\
& $21^{a}$ & 12.65 & 3.96 \\
FSRQ & 49 & 16.24 & 3.37 \\
BLO & 13 & 10.29 & 5.24 \\
GAL & 5 & 1.82 & 15.52 \\
ALL & 67 & 13.96 & 3.81 \\
\hline
\end{tabular}

${ }^{a}$ Excluding outliers $0923+392$ and $1730-130$.

for which $\Gamma_{\mathrm{var}}=37.8$. This source has also been classified as a HPQ (e.g. Véron-Cetty \& Véron 2006) and therefore we ran the $\mathrm{KW}$-analysis again by moving this source into the HPQ class. In this case also BLOs and LPQs differ significantly from each other with a $96 \%$ confidence. We must note that in our samples of 13 to 26 objects the significance of differences can depend on the classification of a single extreme source. However, a clear result is that the BLOs and the quasars differ from each other with BLOs having slower jets (FSRQs and BLOs differ significantly with a $99 \%$ confidence if $1823+568$ is classified as a BLO and with a $99.9 \%$ confidence if $1823+568$ is classified as a HPQ). This result is in accordance with several earlier but more indirect estimates of jet speeds. Similar results have also been obtained with simulations (Hughes et al. 2002).

When $\theta_{\text {var }}$ is studied, the distributions overlap even more and $\mathrm{KW}$-analysis shows that GALs and BLOs differ from other source classes with a $95 \%$ confidence. Also, if the differences between FSRQs and BLOs are studied, they differ from each other significantly with a $99 \%$ confidence with BLOs having larger viewing angles.

We compared our new Lorentz factors with the ones from LV99. There are 38 sources in common in our samples, and in Fig. 9 we can see that the new $\Gamma_{\text {var }}$ are about twice as large as in LV99. This is mainly because also the apparent speeds used in our analysis are about twice as large as in LV99, where they were collected from the literature. We should now have better and more homogeneous estimates of $\beta_{\text {app }}$ which should make our new estimates more accurate. The correlation between our $\Gamma_{\text {var }}$ and those from LV99 is only $r=0.37$ ( $p=0.0113$ ) when the outlier $(0923+392)$ is not included (the other outlier $1730-130$ is not included in the sample of LV99). When we compare our $\Gamma_{\text {var }}$ with Lorentz factors estimated in Jorstad et al. (2005) we find a good correlation of $r=0.50(p=0.0281)$ which gives credibility to our new estimates. Also the distribution is similar with quasars having the highest Lorentz factors, BLOs being in between and GALs having the smallest Lorentz factors. We have only 12 sources in common with the sample of Britzen et al. (2007) and we find no correlation between our Lorentz factors even if we leave out their outlier source $0016+731$ for which they determine a Lorentz factor of 860 . The differences are probably due to their much lower values of $\beta_{\text {app }}$ and differences in the Doppler boosting factors.

When studying the viewing angles, the difference between our new and LV99 values is not as large and the correlation is $\operatorname{good}(r=0.51 p=0.0005)$. The correlation between our values and Jorstad et al. (2005) values is also very good with $r=0.59$ $(p=0.0105)$. Again there are no significant correlations between $\theta_{\text {var }}$ and values from Britzen et al. (2007).

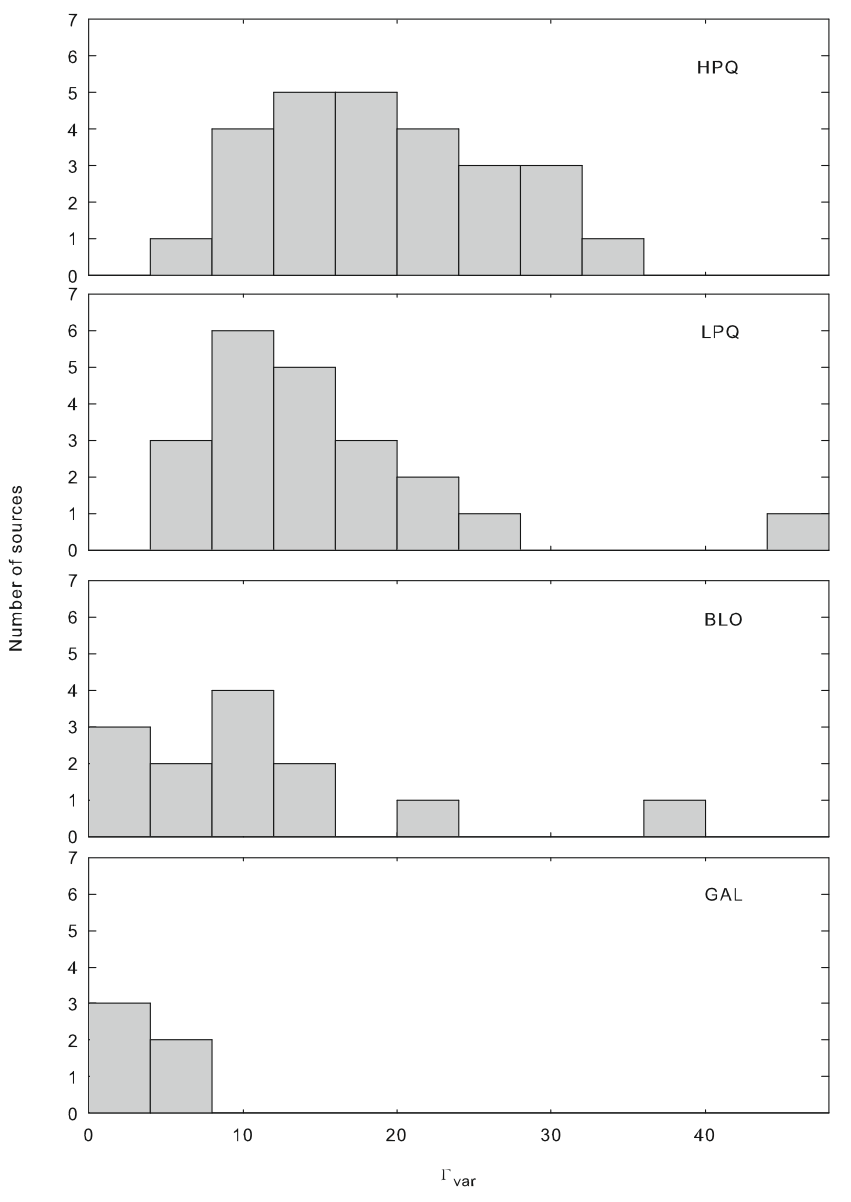

Fig. 7. Distribution of $\Gamma_{\mathrm{var}}$ of the fastest flare in each source (excluding the outlier LPQs 0923+392 and 1730-130).

\section{Discussion}

Although the Doppler boosting factors have remained on the average almost identical (cf. Fig. 4) even though we now have ten more years of data, there is a factor of two difference in the Lorentz factors when comparing our new results to LV99. This is mainly due to twice as fast apparent speeds in our new analysis. In LV99 the $\beta_{\text {app }}$ values are mainly from Vermeulen \& Cohen (1994), in which the values were gathered from the literature. This means that the speeds were obtained in the 1980s and early 1990s from a variety of observing programmes and frequencies, mainly at $5 \mathrm{GHz}$. We use $15 \mathrm{GHz}$ MOJAVE data from 1994 up to September 2008, giving a far more uniform dataset. We also note that higher frequency VLBI observations often tend to give higher apparent speeds as can be seen by comparing, e.g., data from Vermeulen \& Cohen (1994), Kellermann et al. (2004), Jorstad et al. (2005), and Britzen et al. (2007), although the reason for this is not clear. The use of a single frequency therefore diminishes the internal scatter.

Long-term observations, both TFD monitoring and VLBI, are essential in understanding source behaviour. We have already shown this in our earlier studies of long-term variability behaviour of these sources (Hovatta et al. 2007; Hovatta et al. $2008 a, b)$. Some sources have changed their behaviour in our TFD observations during the past 10 years. Similar changes can be seen in VLBI sources over a long time, such as the position swings seen, for example, in 3C 273 (Savolainen et al. 2006) and NRAO 150 (Agudo et al. 2007). We emphasise that our way to estimate the Lorentz factors and viewing angles is as good as can 


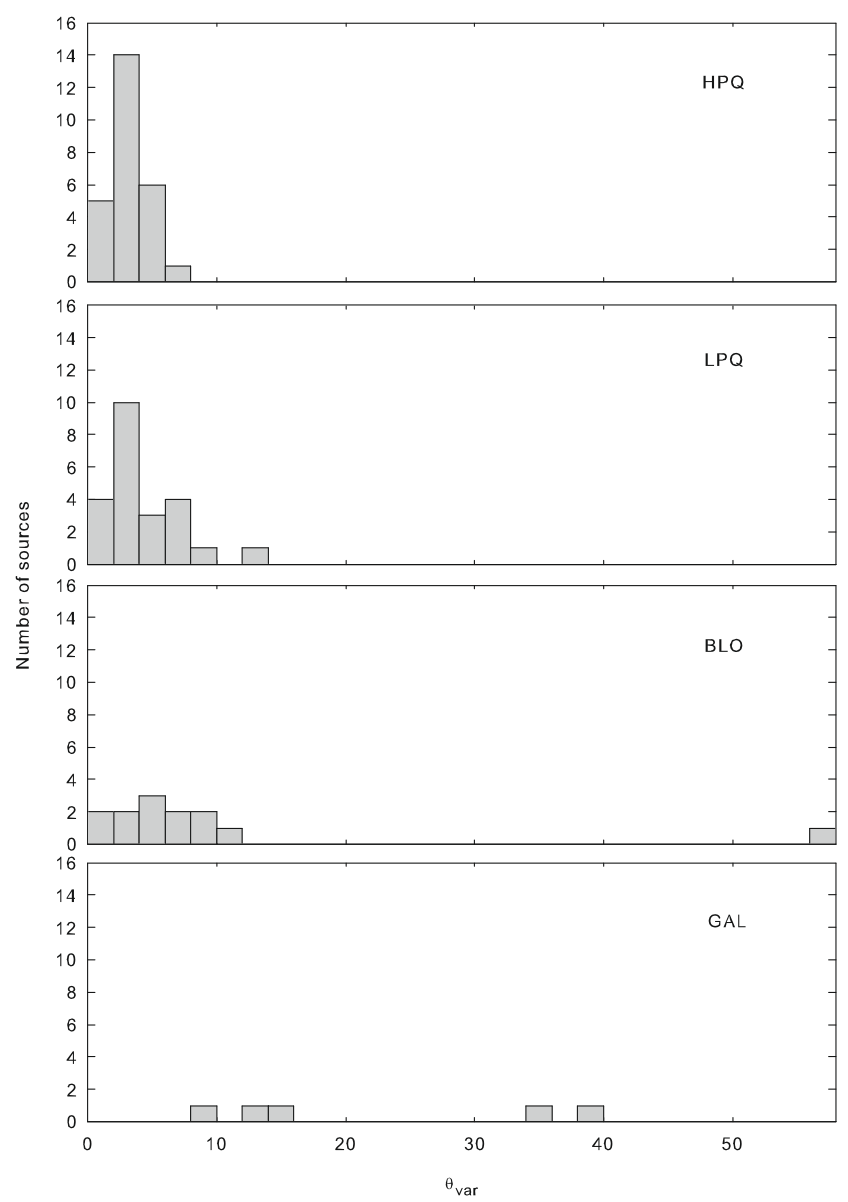

Fig. 8. Distribution of $\theta_{\text {var }}$ of the fastest flare in each source.

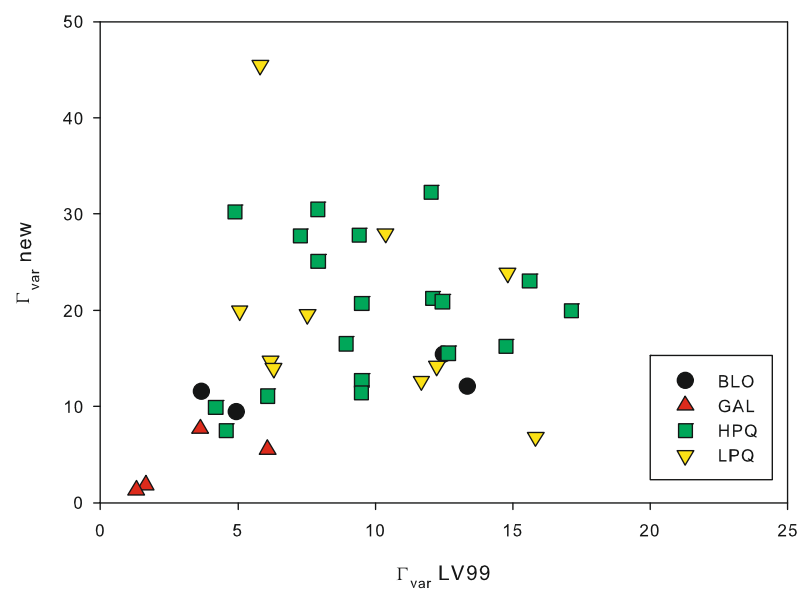

Fig. 9. Correlation between Lorentz factors from LV99 and the new values (excluding the outlier source $0923+392$ with a Lorentz factor of 216 in the new analysis).

be, when we are characterising a complex, changing jet with just two parameters, both assumed to be constant.

In addition to having twice as high apparent speeds in general, there are also some extreme sources showing very high apparent motion. While in LV99 the highest apparent speed was 14.9 , we now have two sources $(0923+392$ and 1730-130) with $\beta_{\text {app }}>35 c$. As a consequence, the Lorentz factors of these sources are also extremely high, $\Gamma_{\mathrm{var}}=216$ for $0923+392$ and $\Gamma_{\mathrm{var}}=65$ for $1730-130$. We also note that if the $\beta_{\mathrm{app}}=45.9$ for

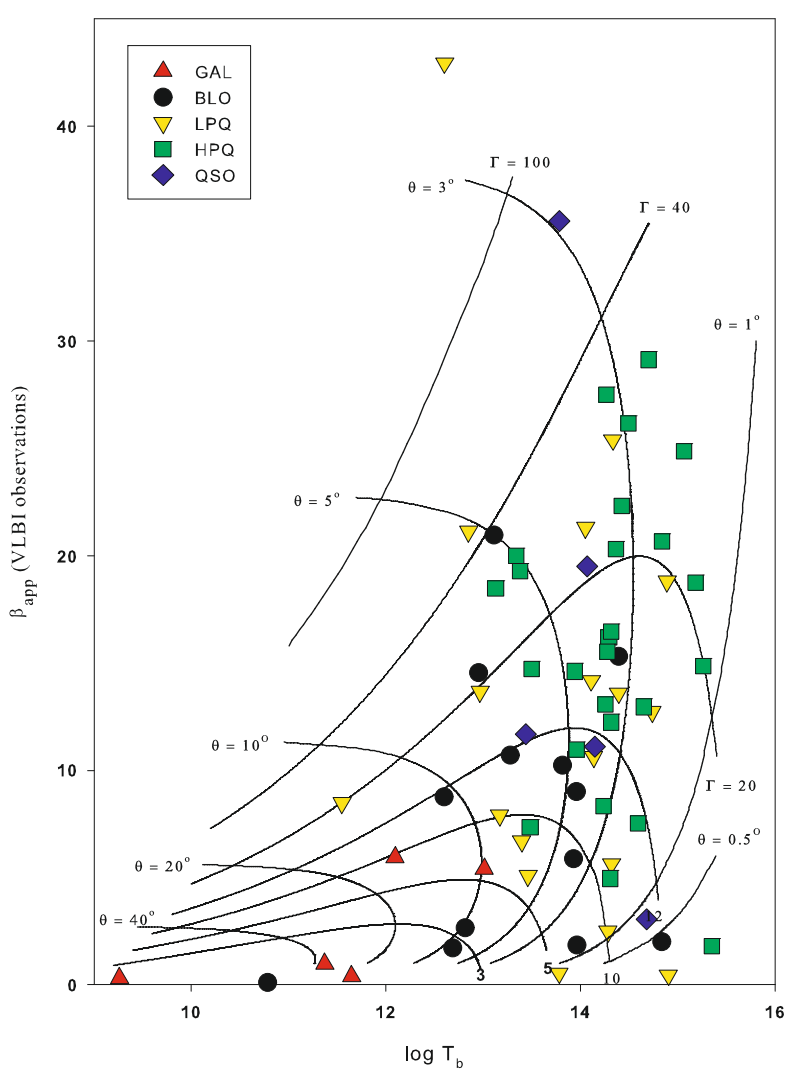

Fig. 10. Observable quantities $\beta_{\text {app }}$ and $\log \left(T_{\mathrm{b} \text {,var }}\right)$ together with intrinsic parameters $\Gamma_{\text {var }}$ and $\theta_{\text {var }}$.

the source 1510-089 from Jorstad et al. (2005) is accepted, our $\log \left(T_{\mathrm{b}, \mathrm{var}}\right)=14.4$ would indicate $\mathrm{a} \Gamma_{\mathrm{var}}=71$. Thus, the existence of a class of very fast jets should perhaps at least be considered as a possibility.

However, at least the $\Gamma_{\text {var }}=216$ seems rather unlikely in view of our current knowledge of the jets in AGN. One alternative explanation is that $0923+392$ has a higher observed brightness temperature than what we have obtained $\left(\log \left(T_{\mathrm{b}, \mathrm{var}}\right)=12.6\right)$ from our monitoring. If we saw changes of about $1 \mathrm{Jy}$ within a time period of a week, the brightness temperature would be of the order of $10^{15} \mathrm{~K}$, which would change the Lorentz factor to a more acceptable value of under 50. Our sampling, like in other monitoring programmes, is too sparse to detect such rapid flares reliably, and therefore we have initiated a denser monitoring schedule for the source. Another possibility is that the source has a complicated internal structure or geometry, such that the TFD variations and the apparent speeds do not refer to the same component (e.g. Alberdi et al. 1993; Fey et al. 1997; Alberdi et al. 2000). However, independent of our $T_{\mathrm{b}, \mathrm{var}}$ estimates, $0923+392$ must have a Lorentz factor of at least 43 as $\Gamma \geq \beta_{\text {app }}$.

In Fig. 10 we have plotted the observable quantities $\beta_{\text {app }}$ and $\log \left(T_{\mathrm{b}, \mathrm{var}}\right)$. We have included curves to mark areas of different $\Gamma_{\mathrm{var}}$ and $\theta_{\mathrm{var}}$. The outlier sources are clearly visible in this plot but otherwise the sources are within rather well-defined limits. Almost all the sources have $\Gamma_{\mathrm{var}}<40$ and $\theta_{\mathrm{var}}<20^{\circ}$. The differences between the source classes are also seen in this plot, with GALs having slow speeds and low brightness temperatures. Using Monte-Carlo simulations, Cohen et al. (2007) find an upper limit for the Lorentz factor to be $\Gamma \approx 32$. This agrees quite well with our results, although in our sample we have five sources with $\Gamma_{\mathrm{var}}$ between 30 and 50 and two quasars with $\Gamma_{\mathrm{var}}>50$. 


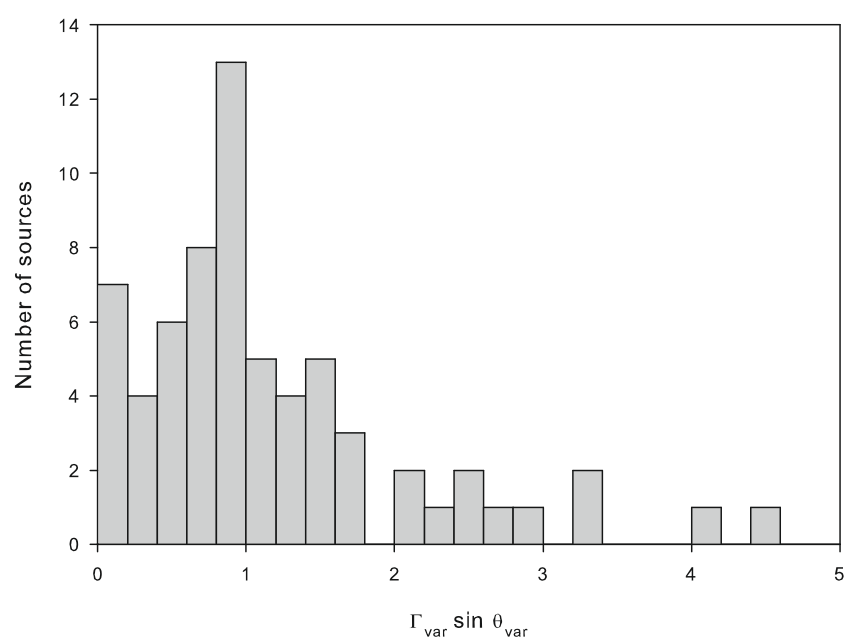

Fig. 11. Distribution of $\Gamma \sin \theta$, excluding the source $0923+392$ with $\Gamma_{\text {var }} \sin \theta_{\text {var }}=10$.

A common assumption is that sources are viewed close to the critical angle $\theta_{\mathrm{c}}=1 / \Gamma$. Figure 11 shows the $\Gamma \sin \theta$ distribution for our sources, which indeed does peak around 1 . However, a number of sources have $\Gamma \sin \theta$ significantly larger or smaller, so that the assumption of $\Gamma \sin \theta=1$ will in many cases lead to false conclusions both for individual sources and for samples. Cohen et al. (2007) have calculated a Monte Carlo simulation for a flux density limited survey (similar to ours), (Fig. 1c in Cohen et al. 2007 , in which the sources with $\Gamma=15$ are plotted), finding a distribution rather similar to our empirical one.

The simplest unification scheme for AGN (e.g. Barthel 1989; Urry \& Padovani 1995) predicts that in all radio quasars (including ordinary quasars and FSRQs) the $\Gamma$ distributions should be similar. Ordinary quasars, on the other hand, should have larger viewing angles than blazars (FSRQs and BLOs). BLOs could also have a different $\Gamma$ distribution because their parent population is different than in quasars. In Fig. 12 we show the Lorentz factors and viewing angles in a polar plot. It is easy to see that almost all the sources are seen at a small viewing angle. What must be kept in mind is that our sample essentially includes the $\sim 100$ brightest northern compact radio sources, so this is as expected for Doppler boosting dominated sources. Although we have divided our quasars into HPQs and LPQs, they all are FSRQs (or blazars), and we do not see ordinary quasars with $\theta_{\text {var }}>20^{\circ}$. Similarly, all our BLOs are radio selected BLOs (RBLs) which should have small viewing angles (Barthel 1989). In addition, the five GALs in our sample are the most compact and variable sources of their type. Figure 13 also shows how the sources at different redshifts are selected due to their Doppler boosting so that small Doppler boosting factors are not seen at high redshifts. These caveats must be kept in mind when comparing our different classes of sources with each other.

The distribution of $\Gamma_{\text {var }}$ agrees well with unification schemes for AGN. Padovani \& Urry (1992) and Urry et al. (1991) calculated beamed luminosity functions for FSRQs and BLOs, and compared them with observed luminosity functions, which were assumed to have the same shape as the intrinsic luminosity function of radio-loud quasars. The validity of this assumption was recently confirmed by using the maximum likelihood method to calculate the intrinsic luminosity functions (Liu \& Zhang 2007; Cara \& Lister 2008). In Padovani \& Urry (1992) the FSRQs were best described with a distribution of $5 \lessgtr \Gamma \lesssim 40$ with an average of $\sim 11$ (using a cosmology with $H_{0}=50 \mathrm{~km} \mathrm{~s}^{-1} \mathrm{Mpc}^{-1}$
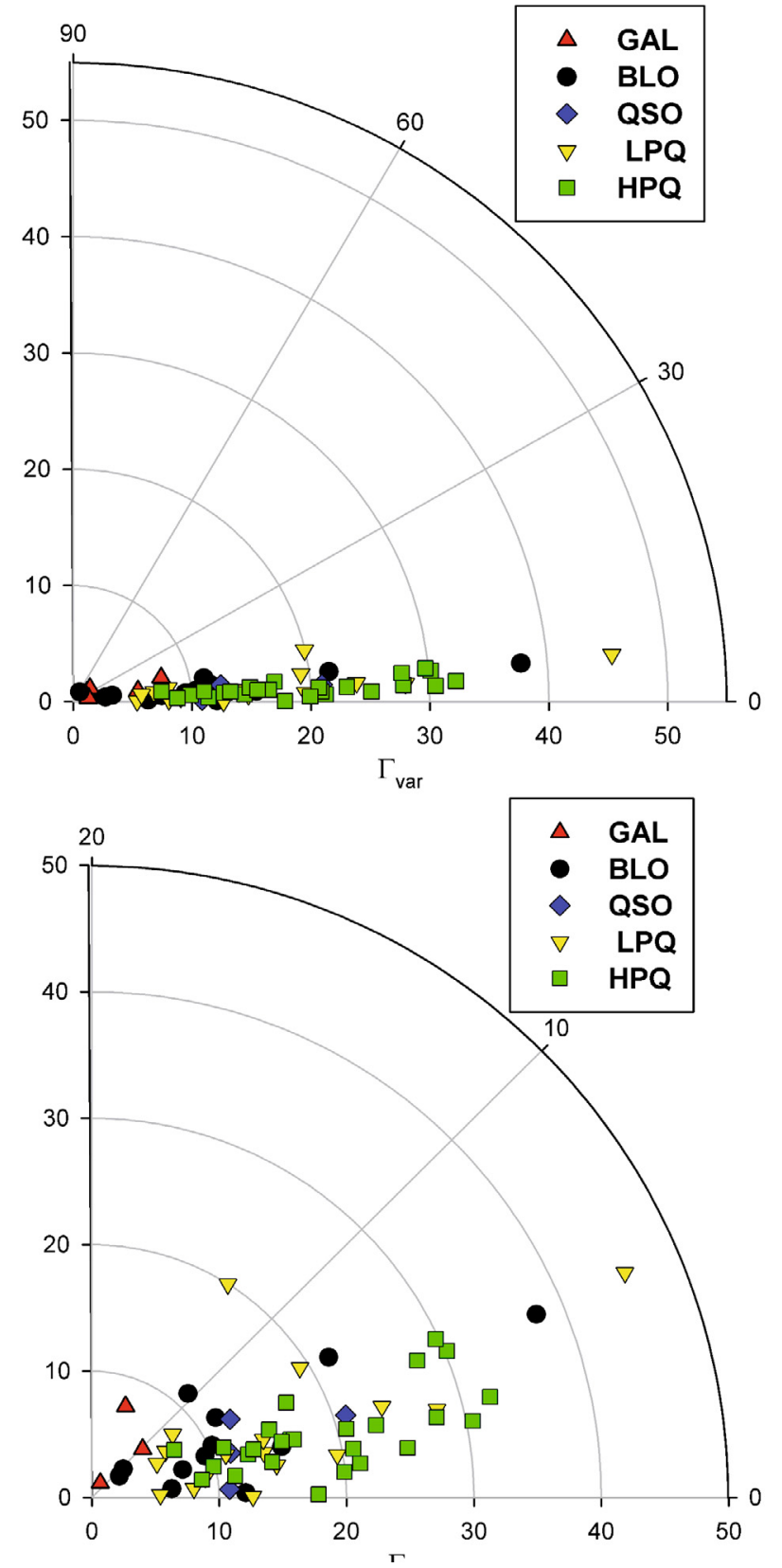

Fig. 12. Polar plot of Lorentz factors against the viewing angles, excluding the outlier sources $0923+392$ and 1730-130. The lower panel shows only the $0^{\circ}$ to $20^{\circ}$ portion of the viewing angles, additionally excluding the galaxies $0007+106$ and $0316+413$, and the BLO $1807+698$.

and $\left.q_{0}=0\right)$. In our sample all the FSRQs have $\Gamma_{\mathrm{var}}>5$ and three have $\Gamma_{\text {var }}>40$. The median $\Gamma_{\text {var }}$ is somewhat bigger than the average from the luminosity functions. In Urry et al. (1991) the BLOs were best described with a distribution of $5 \lesssim \Gamma \lesssim 35$ with an average of $\sim 7$. Again we have only three BLOs with $\Gamma_{\text {var }}<5$ and one BLO (1823+568, also classified as HPQ) with $\Gamma_{\text {var }}>35$. Our median is also only slightly bigger. In addition they made another fit for the BLOs with a distribution of $2 \lesssim \Gamma \lesssim 20$ which also fit the data well. In this case only one BLO $(1807+698$ with $\left.\Gamma_{\mathrm{var}}=1.0\right)$ would be below the lower limit and there would be two BLOs with $\Gamma_{\text {var }}>20$. Their calculations also set a value for the critical viewing angle in which all the FSRQs and BLOs should be seen. For FSRQs the angle is $14^{\circ}$, and all our quasars are within this limit. For BLOs the critical angle is either $11^{\circ}$ 


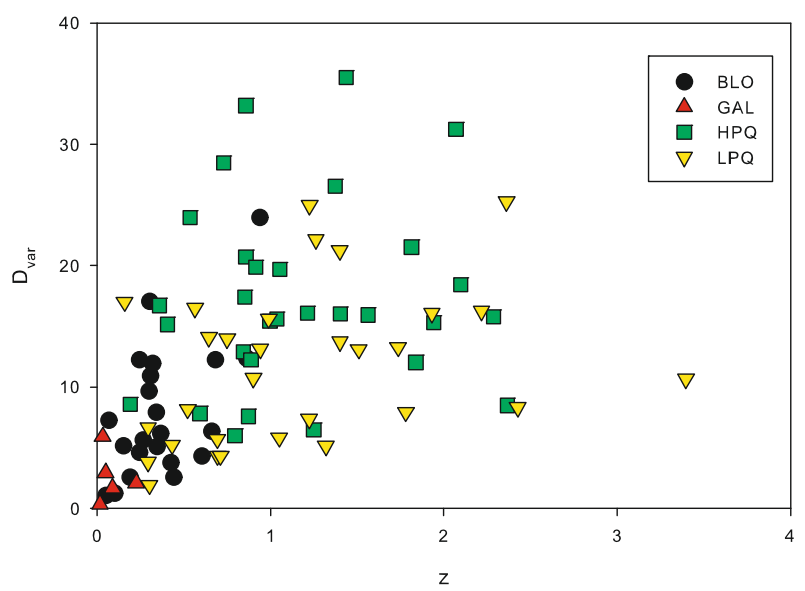

Fig. 13. $D_{\text {var }}$ against the redshift.

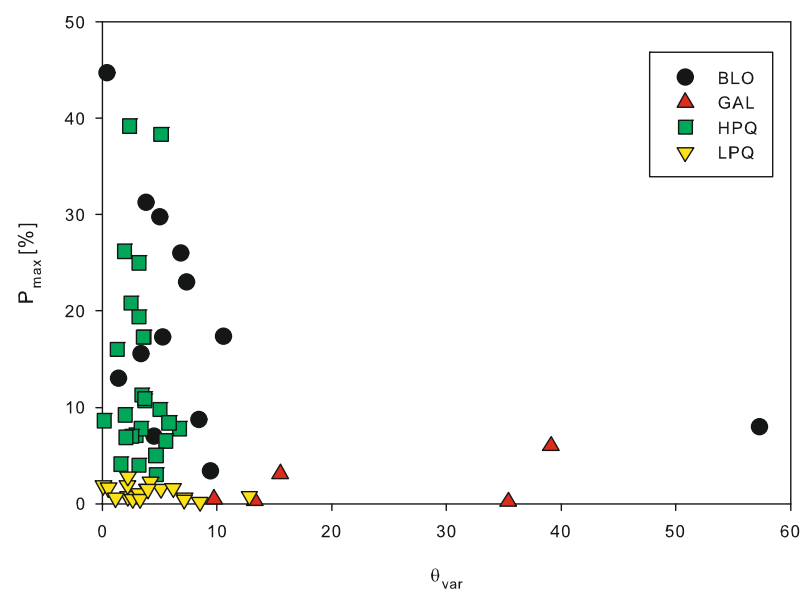

Fig. 14. Maximum optical polarisation against the viewing angle.

or $19^{\circ}$ depending on the model. In both cases only one BLO $\left(1807+698\right.$ with $\theta_{\mathrm{var}}=57^{\circ}$, which is also the nearest BLO in our sample) would have a viewing angle outside the limit.

As expected with sources with small viewing angles, distributions of LPQs and HPQs overlap and one should not identify LPQs as ordinary quasars. In Fig. 14 we show the maximum optical polarisation against the viewing angle for all the sources for which we could find polarisation information in the literature $\left(P_{\max }\right.$ and its reference for all the sources are shown in Table 1). Most of the sources seem to be within an envelope with $P_{\max }$ decreasing as $\theta_{\text {var }}$ increases. The situation may be similar as in Seyfert galaxies with an obscuring torus (e.g. Schmitt et al. 2001). When the viewing angle is small, we can see deeper into the jet and are more likely to see the source as more optically polarised. Also, it has been shown that both the optical and the radio polarisation originate from transverse shocks located very close to the base of the jet (Lister \& Smith 2000). Therefore the range of polarisation variability is much higher for sources with a viewing angle of a few degrees than for sources with $\theta_{\mathrm{var}}=10^{\circ}-20^{\circ}$. In Fig. 14 it is also possible to see that the definition $P_{\max }=3 \%$ for an object to be classified as highly polarised is somewhat artificial. We believe that with a larger number of polarisation observations, a considerable fraction of the LPQ population could, at times, show polarisation exceeding 3\%, as expected from blazar-type AGN. Since there usually is only a small number of polarisation observations, the likelihood of observing high polarisation increases with decreasing viewing angle. However, as has been pointed out by Lister \& Smith (2000), there is a physical difference between at least some HPQs and LPQs, especially in the magnetic field structure of their inner jets.

Figure 14 hints that the transition from blazar-type AGN to ordinary quasars may occur around a viewing angle of $15^{\circ}$ to $20^{\circ}$, presumably corresponding to the half opening angle of an AGN obscuring torus, and in agreement with, e.g., estimates from luminosity functions and source counts. As our sample does not contain any ordinary quasars, no definite conclusions can be drawn.

\section{Conclusions}

We have decomposed flux curves of 87 sources into exponential flares and using the fits calculated the variability brightness temperature and Doppler boosting factor for each source. In addition we used new MOJAVE observations of apparent jet speeds to calculate the variability Lorentz factors and viewing angles. We have compared our results with LV99, in which the parameters were determined in a similar way. In our new analyses we have used almost 15 years more data and the estimates should be more accurate. Our main conclusions can be summarised as follows.

1. The variability Doppler boosting factors have remained on the average almost identical compared to LV99. All the quasars are Doppler-boosted and they are in general more boosted than the BLOs or GALs.

2. The Lorentz factors of our new analyses are about twice as large as in LV99. The difference can be explained with twice as large apparent VLBI speeds in our new analyses. The BLOs have slower jets compared to quasars $\left(\Gamma_{\mathrm{BLO}}=10.3\right.$, $\Gamma_{\mathrm{FSRQ}}=16.2$ and $\Gamma_{\mathrm{GAL}}=1.8$ ).

3. Two sources, $0923+392$ and 1730-130, have extreme Lorentz factors (216 and 65, respectively). Either these Lorentz factors are real, or the sources exhibit so rapid flares that the fast variations have remained undetected in the monitoring programmes. We are studying the second possibility with dense observations of $0923+392$. A third possibility is that the sources have a structure too complex for our method.

4. Almost all the sources in our sample are seen in a small viewing angle of less than 20 degrees $\left(\theta_{\mathrm{BLO}}=5.2^{\circ}, \theta_{\mathrm{FSRQ}}=3.4^{\circ}\right.$ and $\theta_{\mathrm{GAL}}=15.5^{\circ}$ ).

5. The viewing angle distribution peaks around $\Gamma \sin \theta=1$, with a distribution similar to that found in simulations.

6. Our results generally follow the predictions of basic unification models for AGN. Based on our results, we cannot separate HPQs and LPQs from each other, and therefore it is well-grounded to treat them as a single group of FSRQs when the jet parameters are considered.

Acknowledgements. We thank M. Lister and the members of the MOJAVE team for providing data in advance of publication. We acknowledge the support of the Academy of Finland (project numbers 212656 and 210338). This research has made use of data from the MOJAVE (Lister and Homan, 2005, AJ, 130, 1389) and $2 \mathrm{~cm}$ Survey (Kellermann et al. 2004, ApJ, 609, 539) programs.

\section{References}

Agudo, I., Bach, U., Krichbaum, T. P., et al. 2007, A\&A, 476, L17 Alberdi, A., Marcaide, J. M., Marscher, A. P., et al. 1993, ApJ, 402, 160 Alberdi, A., Gómez, J. L., Marcaide, J. M., Marscher, A. P., \& Pérez-Torres, M. A. 2000, A\&A, 361, 529

Angel, J. R. P., \& Stockman, H. S. 1980, ARA\&A, 18, 321

Barthel, P. D. 1989, ApJ, 336, 606 
Britzen, S., Brinkmann, W., Campbell, R. M., et al. 2007, A\&A, 476, 759 Britzen, S., Vermeulen, R. C., Campbell, R. M., et al. 2008, A\&A, 484, 119 Cara, M., \& Lister, M. L. 2008, ApJ, 674, 111

Cohen, M. H., Lister, M. L., Homan, D. C., et al. 2007, ApJ, 658, 232

Fey, A. L., Eubanks, M., \& Kingham, K. A. 1997, AJ, 114, 2284

Ghisellini, G., Padovani, P., Celotti, A., \& Maraschi, L. 1993, ApJ, 407, 65

Guerra, E. J., \& Daly, R. A. 1997, ApJ, 491, 483

Guijosa, A., \& Daly, R. A. 1996, ApJ, 461, 600

Homan, D. C., Ojha, R., Wardle, J. F. C., et al. 2001, ApJ, 549, 840

Homan, D. C., Kovalev, Y. Y., Lister, M. L., et al. 2006, ApJ, 642, L115

Hovatta, T., Tornikoski, M., Lainela, M., et al. 2007, A\&A, 469, 899

Hovatta, T., Lehto, H. J., \& Tornikoski, M. 2008a, A\&A, 488, 897

Hovatta, T., Nieppola, E., Tornikoski, M., et al. 2008b, A\&A, 485, 51

Hughes, P. A., Miller, M. A., \& Duncan, G. C. 2002, ApJ, 572, 713

Impey, C. D., \& Tapia, S. 1990, ApJ, 354, 124

Impey, C. D., Lawrence, C. R., \& Tapia, S. 1991, ApJ, 375, 46

Impey, C. D., Bychkov, V., Tapia, S., Gnedin, Y., \& Pustilnik, S. 2000, AJ, 119, 1542

Jorstad, S. G., Marscher, A. P., Mattox, J. R., et al. 2001, ApJS, 134, 181

Jorstad, S. G., Marscher, A. P., Lister, M. L., et al. 2005, AJ, 130, 1418

Jorstad, S. G., Marscher, A. P., Stevens, J. A., et al. 2007, AJ, 134, 799

Kellermann, K. I., Lister, M. L., Homan, D. C., et al. 2004, ApJ, 609, 539

Kovalev, Y. Y., Kellermann, K. I., Lister, M. L., et al. 2005, AJ, 130, 2473

Lähteenmäki, A., \& Valtaoja, E. 1999, ApJ, 521, 493, LV99

Lähteenmäki, A., Valtaoja, E., \& Wiik, K. 1999, ApJ, 511, 112

Lister, M. L., \& Smith, P. S. 2000, ApJ, 541, 66

Liu, Y., \& Zhang, S. N. 2007, ApJ, 667, 724

Moore, R. L., \& Stockman, H. S. 1984, ApJ, 279, 465

Nieppola, E., Tornikoski, M., Lähteenmäki, A., et al. 2007, AJ, 133, 1947
Padovani, P., \& Urry, C. M. 1992, ApJ, 387, 449

Piner, B. G., Mahmud, M., Fey, A. L., \& Gospodinova, K. 2007, AJ, 133, 2357

Punsly, B. 1996, ApJ, 473, 152

Readhead, A. C. S. 1994, ApJ, 426, 51

Salonen, E., Teräsranta, H., Urpo, S., et al. 1987, A\&AS, 70, 409

Savolainen, T., Wiik, K., Valtaoja, E., Jorstad, S. G., \& Marscher, A. P. 2002, A\&A, 394, 851

Savolainen, T., Wiik, K., Valtaoja, E., \& Tornikoski, M. 2006, A\&A, 446, 71

Schmitt, H. R., Antonucci, R. R. J., Ulvestad, J. S., et al. 2001, ApJ, 555, 663

Sluse, D., Hutsemékers, D., Lamy, H., Cabanac, R., \& Quintana, H. 2005, A\&A, 433, 757

Smith, P. S., Balonek, T. J., Heckert, P. A., Elston, R., \& Schmidt, G. D. 1985, AJ, 90, 1184

Stickel, M., \& Kuehr, H. 1994, A\&AS, 105, 67

Taylor, G. B., Vermeulen, R. C., Readhead, A. C. S., et al. 1996, ApJS, 107, 37

Teräsranta, H., Tornikoski, M., Valtaoja, E., et al. 1992, A\&AS, 94, 121

Teräsranta, H., Tornikoski, M., Mujunen, A., et al. 1998, A\&AS, 132, 305

Teräsranta, H., Achren, J., Hanski, M., et al. 2004, A\&A, 427, 769

Teräsranta, H., Wiren, S., Koivisto, P., Saarinen, V., \& Hovatta, T. 2005, A\&A, 440, 409

Urry, C. M., \& Padovani, P. 1995, PASP, 107, 803

Urry, C. M., Padovani, P., \& Stickel, M. 1991, ApJ, 382, 501

Valtaoja, E., Lähteenmäki, A., Teräsranta, H., \& Lainela, M. 1999, ApJS, 120, 95

Vermeulen, R. C., \& Cohen, M. H. 1994, ApJ, 430, 467

Véron-Cetty, M.-P., \& Véron, P. 2006, A\&A, 455, 773

Wills, B. J., Wills, D., Breger, M., Antonucci, R. R. J., \& Barvainis, R. 1992, ApJ, 398, 454

Wright, E. L. 2006, PASP, 118, 1711 